\title{
Treatment of Class III Malocclusion at Later Stage of Growth
}

\author{
${ }^{1}$ Suruchi Jatol-Tekade, ${ }^{2}$ Satyajit A Tekade, ${ }^{3}$ Sachin C Sarode, ${ }^{4}$ Gargi S Sarode, ${ }^{5}$ Shankargouda Patil
}

\section{ABSTRACT}

Aim: The aim of the study is to try growth modification in late pubertal growth.

Introduction: In the given case report, clinician tried tandem appliance for correcting skeletal class III malocclusion in a patient on the verge of growth completion.

Case report: A 12-year-old female patient with maxillary deficiency was treated with growth modulation therapy for maxillary advancement. Residual growth potential was fully utilized and facial harmony was established for future.

Conclusion: Class III malocclusion in adolescents is challenging to treat. Incidence of class III malocclusion is $<5 \%$ among Indian population, and lack of awareness creates difficulties in its treatment options. If a growing patient reports on time with the complaint of sunken face appearance or cross-bite, the clinician may treat the patient very well with relevant treatment options. Because of lack of awareness, the patient with Class III malocclusion reported after completion of growth. This situation reduces available treatment modalities.

Clinical significance: We are reporting a case of a female patient with class III malocclusion at the end of growth completion. Treatment modalities tried at the end of growth were found to be effective. Chin cap was given to the patient to prevent relapse from any possible remaining growth of mandible.

Keywords: Class III malocclusion, Reverse pull headgear, Tandem appliance.

How to cite this article: Jatol-Tekade S, Tekade SA, Sarode SC, Sarode GS, Patil S. Treatment of Class III Malocclusion at Later Stage of Growth. World J Dent 2017;8(5):417-421.

Source of support: Nil

Conflict of interest: None

\footnotetext{
${ }^{1}$ Department of Orthodontics \& Dentofacial Orthopedics, Modern Dental College \& Research Centre, Indore, Madhya Pradesh India

${ }^{2}$ Department of Oral \& Maxillofacial Pathology, Modern Dental College \& Research Centre, Indore, Madhya Pradesh, India

${ }^{3,4}$ Department of Oral Pathology \& Microbiology, Dr. D. Y. Patil Dental College \& Hospital, Dr. D. Y. Patil Vidyapeeth, Pune Maharashtra, India

${ }^{5}$ Division of Oral Pathology, Department of Maxillofacial Surgery and Diagnostic Sciences, College of Dentistry, Jazan University Jazan, Kingdom of Saudi Arabia

Corresponding Author: Satyajit A Tekade, Department of Oral \& Maxillofacial Pathology, Modern Dental College \& Research Centre, Indore, Madhya Pradesh, India, e-mail: suruchitekade@ gmail.com
}

\section{INTRODUCTION}

Class III malocclusion may present clinically as concave profile or straight profile with anterior divergence, midface deficiency resulting in sunken appearance, relative mandibular prognathism, prominent chin, with anterior cross-bite or edge-to-edge relation, and narrow maxillary arch with or without posterior cross-bite. ${ }^{1}$ Class III malocclusion gives senile look to young adults. Parents generally do not notice the deformity until adulthood, as it worsens with age. This may be because in Indian population, incidence of class III malocclusion is just 0.3 to $3.4 \%^{2}$; so, it goes unnoticed until the disturbed position of maxilla results in facial imbalance. Dentofacial deformities are best treated in growing age to achieve proper facial profile and function.

The early orthopedic intervention for class III deformity, before growth spurt, modifies the orofacial growth and development, thereby, providing facial muscular balance and preventing a future invasive treatment by increasing the stability. ${ }^{3}$ Early treatment allows greater opportunity to modify craniofacial skeletal growth, improved confidence of the child, and parental contentment. Proper modulation of growth during growing period prevents less extensive therapy required later and reduces the possibility of iatrogenic tooth damage, such as parasthesia, root resorption, decalcification, and trauma. ${ }^{4}$ To improve growing facial balance, class III malocclusion should be treated as soon as it is reported. This article presents such a case report treated with tandem protraction appliance relatively at later stage of growth. However, comparing the other advantages of growth modulation over surgical treatment, the clinician took a chance to improve facial harmony of this patient, which resulted positively.

\section{CASE REPORT}

A 12-year-old female patient reported with a chief complaint of crowding in upper front teeth. The patient had cleft lip without cleft palate and was treated for it at age of 10 months. Clinical examination revealed slight midfacial deficiency and straight profile with anterior divergence, characterizing mild class III malocclusion (Fig. 1). There was no occlusal interference or deviation of mandible on closure. Investigations confirmed class III malocclusion. 

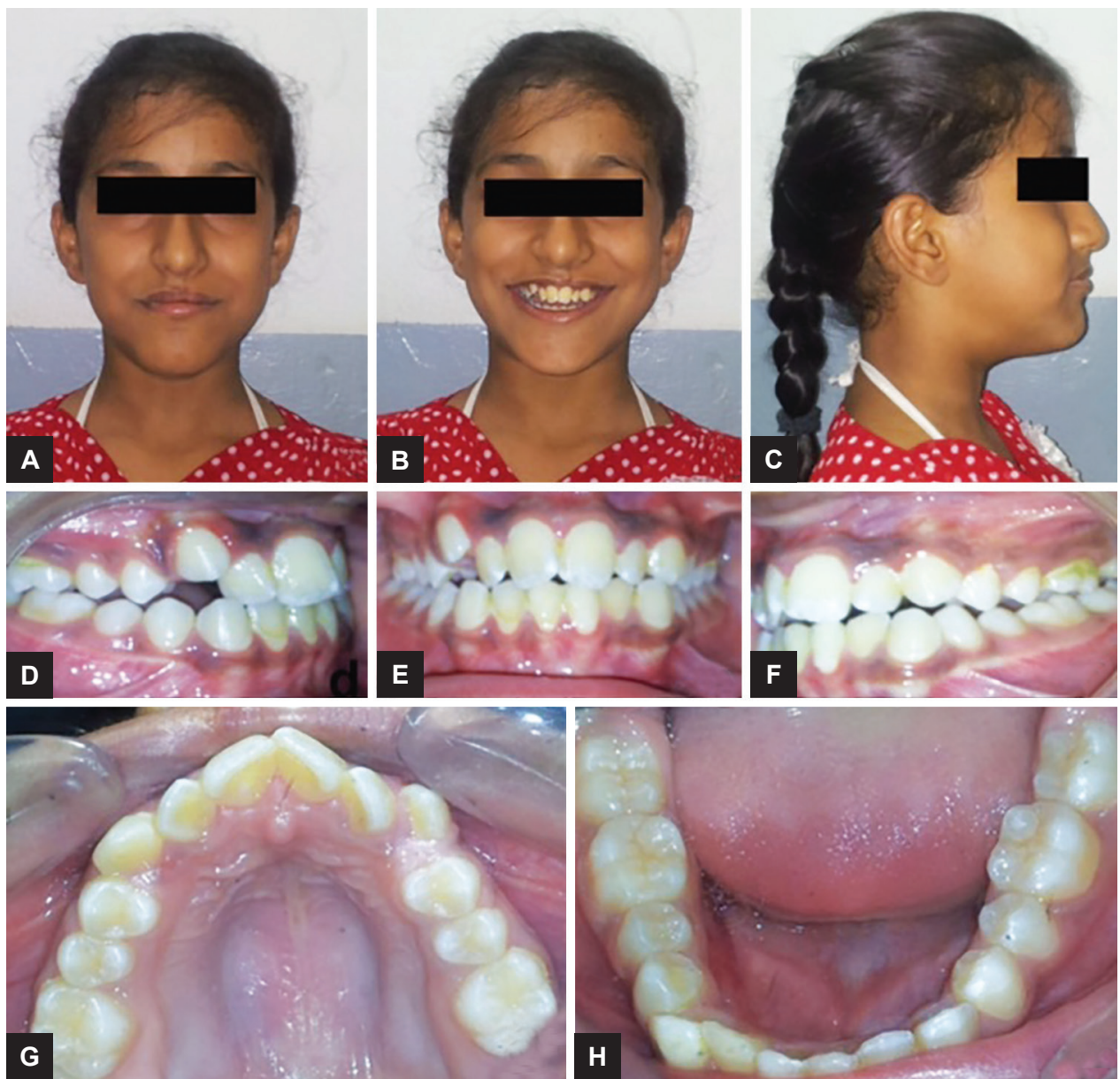

Figs 1A to H: Pretreatment images: (A) extraoral front view; (B) extraoral smiling view; (C) extraoral profile view; $(D)$ intraoral—in occlusion right lateral; $(E)$ intraoral-in occlusion front; $(F)$ intraoral—in occlusion left lateral; $(\mathrm{G})$ upper arch; and $(\mathrm{H})$ lower arch

Intraorally, she had super class I molar relationship on right side and class III molar relation on left side. Class I canine relation on left side, and right canine was not fully erupted. The panoramic radiograph showed no missing teeth or pathologies. Cephalometric analysis indicated a skeletal class III maxilla-mandibular relationship due to short maxillary length (the linear measurement between point $\mathrm{N}$ perpendiculars to point $\mathrm{A}$ was $-4 \mathrm{~mm}$ ) along with a vertical growth pattern with upright upper incisors. However, short maxilla could not create normal overjet and overbite resulting in edge-to-edge bite, which otherwise could have landed in reverse overjet, if not treated in time. Patient and parents were made aware of the situation and options were available for treating such conditions. Rather than waiting until adulthood and undergoing surgery, they opted for a conventional way.

The upper first permanent molars were banded, and a modified tandem appliance (with hyrax expansion screw) was fabricated and inserted (Fig. 2). The girl was on regular follow-up. The anterior edge-to-edge bite was corrected after 9 months of active treatment. The profile also showed a significant improvement (Fig. 3). The appliance was removed at a total of 18 months after
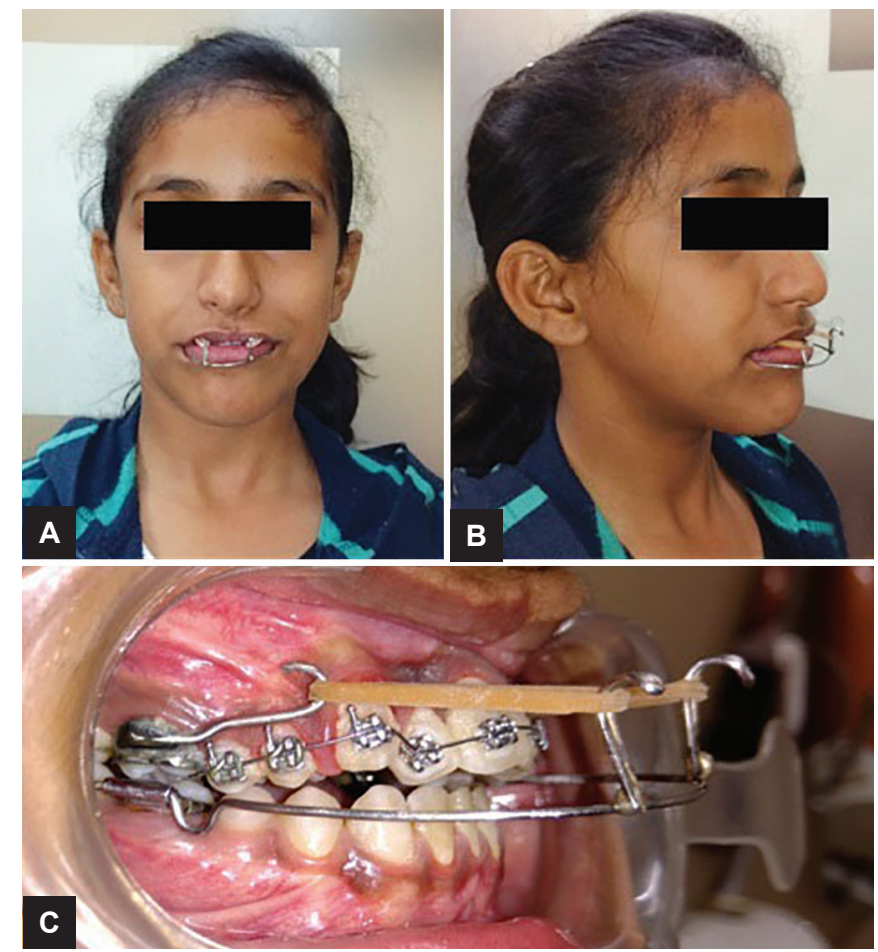

Figs $2 \mathrm{~A}$ to $\mathrm{C}$ : Tandem maxillary protraction appliance in patient: (A) Extraoral front view; (B) extraoral profile view; and (C) intraoral-in occlusion right lateral 


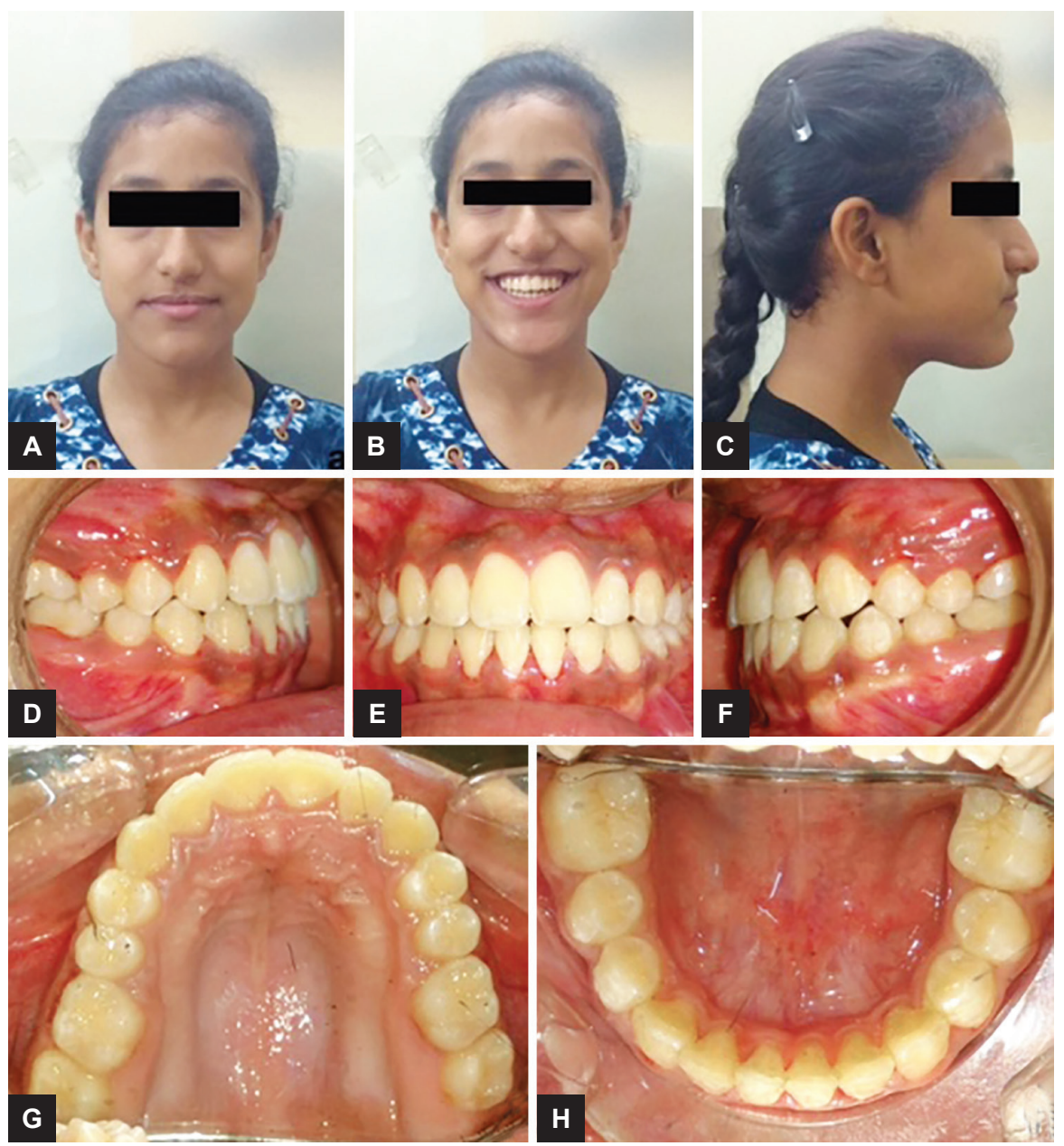

Figs $3 \mathbf{A}$ to $\mathbf{H}$ : Posttreatment images: (A) Extraoral front view; (B) extraoral smiling view; (C) extraoral profile view; (D) intraoral-in occlusion right lateral; $(E)$ intraoral-in occlusion front; $(F)$ intraoral—in occlusion left lateral; $(G)$ upper arch; and $(H)$ lower arch

active treatment phase. Cephalometric evaluation and superimposition revealed skeletal improvement.

\section{Treatment Objectives}

Patient's cephalometric analysis showed class III skeletal pattern. The treatment objective was to achieve class I pattern utilizing left overgrowth potential with the help of maxillary protraction appliance. The patient was having an edge-to-edge bite, which could be traumatic later on. The second objective was to achieve positive overjet and overbite, which will serve as a lock for growing mandible. The third objective was to achieve class I canine relationship. In addition, more than anything else, obtaining pleasant soft tissue profile was clinician's esthetic goal. Retention phase is critical in growing class III patients. Measures to prevent relapse were taken as chin cap wear.

\section{Treatment Plan}

Orthopedic treatment was planned to induce remaining growth potential of maxilla and improve facial esthetics.
The facemask could have been planned, but because it requires lot of patient compliance than tandem appliance, it was preferred over facemask. Hence, rapid maxillary expansion (RME) to loosen the maxilla from circummaxillary sutures ${ }^{5,6}$ and sagittal maxillary advancement with tandem appliance to correct class III skeletal malocclusion was done. Fixed orthodontic mechanotherapy with pre-adjusted edgewise appliance was carried out for the final detailing of occlusion.

\section{Treatment Progress}

The patient was treated with hyrax expansion screw and tandem appliance. The upper assembly comprised a hyrax expansion screw with first molars and first premolars banded. The protraction hooks in the maxilla were placed distal to the canines, in the premolar region so that the elastic force passes exactly through the center of resistance of the maxilla, as no rotation of occlusal or palatal plane was needed in this case. On both sides, a force of 400 to $450 \mathrm{~g}$ was applied bilaterally for 14 to 16 hours per day. Both arches were strapped up for alignment 
and leveling. Final finishing was done. Full expression of tip and torque was achieved until $0.021 \times 0.025$-inch stainless steel wire fitted snuggly in both arches.

\section{Treatment Results}

The patient was assessed every month until satisfactory results were achieved. Progress records were taken after 18 months of active treatment. Favorable growth of the deficient maxilla and normal overjet overbite were achieved.

Postprotraction cephalometric tracings revealed a forward movement of maxilla. The ANB angle increased from $-1^{\circ}$ to $2^{\circ}$. Point $\mathrm{N}$ perpendicular to $(\perp)$ point $\mathrm{A}$ increased by $3 \mathrm{~mm}$. Minor labial tipping of the upper incisors was also achieved. Upper 1 to NA distance increased from 4 to $8 \mathrm{~mm}$. The point $\mathrm{A}$ also shifted anteriorly by $3 \mathrm{~mm}$ approximately. Lower incisors became more upright.

The patient was given chin cap for retention of the achieved results for another 6 months. The patient is on regular follow-up.

\section{DISCUSSION}

The orthodontic treatment success with a developing class III skeletal discrepancy depends on the residual growth potential of individual and timing of growth modulation. In patients with moderate-to-severe class III discrepancy, the decision of whether to treat early or to wait is difficult until the growth completion. Kluemper and Spalding ${ }^{7}$ advanced our thinking about orthopedic treatment timing of older children. Skeletal class III patients with a maxillary deficiency gain considerable results from early orthopedic treatment. However, growth modulation may produce more favorable changes for older children and adolescents than thought in the past. Orthopedic correction of the mild-to-moderate skeletal class III malocclusion should also include regular followup and growth evaluation. This should be done to avoid dental compensations with small skeletal change that will tax surgical intervention in the future. Rapid maxillary expansion appears to be effective and stable before late puberty, a stage of development when ossification of the maxillary sutures is more advanced. He also added the importance of timing for palatal expansion according to specific needs of each patient. ${ }^{7}$ Considering this fact, the clinician tried to apply orthopedic force in this case and to use remaining growth potential. Although previous studies ask for early intervention, orthopedic force was tried in later stage of growth in a female patient. A comparative study of maxillary advancement in deciduous dentition and mixed dentition by Kajiyama et $\mathrm{l}^{8}$ in
2004 has shown maximum effect in deciduous dentition. Takada et $\mathrm{al}^{9}$ reported that the maxillary advancement with protraction was more stable before or during the acceleration of a child's pubertal growth spurt. Baccetti et $\mathrm{al}^{10}$ reported that treatment of class III malocclusion by maxillary protraction with expansion was effective in the maxilla only when it was done before the peak of pubertal growth spurt. For treating deficient maxilla in class malocclusion, a combination of maxillary protraction and RME has been used. ${ }^{6}$ The RME is done to disengage the maxilla from circummaxillary sutures and to push forward movement of the maxilla. ${ }^{11}$ In this case report, the expansion of maxilla helped disarticulate it and gave us positive results by bringing the maxilla forward. Postprotraction radiographs showed increase in maxillary length, but no change in facial height or mandibular angle. A noticeable increase in ANB after tandem appliance application was due to the forward movement of the maxilla. Although facemask appliances bring the same orthopedic effects, they ask for more patient compliance. They are neither esthetic nor comfortable due to their appearance, discomfort, and skin irritation from the anchorage pads. Chun et $\mathrm{a}^{12}$ defined tandem appliance as a comfortable and more esthetic device than conventional appliances because it is removable and more of intraoral type than comparatively disfiguring facemask appliance. Thus, it can be used for more duration per day. Tortop et $\mathrm{al}^{13}$ compared the effects of the modified tandem traction bow appliance and the facemask in patients being treated for class III malocclusion and found both appliances were effective. In the present case, tandem appliance was used considering the patient compliance and treatment results. Results were satisfying to the parents and patient herself. The patient reported at a very crucial time of her pubertal growth spurt and was treated for class III malocclusion. Positive overjet will prevent overgrowth of mandible and balanced facial profile will further boost her self-esteem in peer-pressured teenage.

Although class III cases are hardly reported at early age in India, awareness about their treatment and positive results is necessary.

\section{CONCLUSION}

The mainstay of all options available for treating developing class III malocclusion is to improve the facial profile and allow the normal growth of the craniofacial bones. Previously, the reporting of class III malocclusion earlier will be the diagnosis, making treatment simpler and faster. Hence, even if a child in late puberty comes with class III malocclusion, clinician can definitely try bone modulation approaches to give better image. 


\section{REFERENCES}

1. Millet D, Wellbury R. Class III malocclusion. In: Orthodontics and Pediatric Dentistry. 1st ed. London: Harcourt Publishers; 2000. p. 48-50.

2. Agarwal SS, Jayan B, Chopra SS. An overview of malocclusion in India. J Dent Health Oral Disord Ther 2015;3(3):00092.

3. Almeida MR, Almeida RR, Oltramari-Navarro PV, Conti AC, Navarro Rde L, Camacho JG. Early treatment of Class III malocclusion: 10-year clinical follow-up. J Appl Oral Sci 2011 Jul-Aug;19(4):431-439.

4. Bishara SE, Justus R, Graber TM. Proceedings of the workshop discussion on early treatment. Am J Orthod Dentofacial Orthop 1998;113:5-6.

5. McNamara JA Jr. An orthopedic approach to the treatment of Class III malocclusion in young patients. J Clin Orthod 1987 Sep;21(9):598-608.

6. Turley PK. Orthopedic correction of Class III malocclusion with palatal expansion and custom protraction headgear. J Clin Orthod 1988 May;22(5):314-325.

7. Kluemper GT, Spalding PM. Realities of craniofacial growth modification. Atlas Oral Maxillofac Surg Clin North Am 2001 Mar;9(1):23-51.
8. Kajiyama K, Murakami T, Suzuki A. Comparison of orthodontic and orthopedic effects of a modified maxillary protractor between deciduous and early mixed dentitions. Am J Orthod Dentofacial Orthop 2004 Jul;126(1):23-32.

9. Takada K, Petdachai S, Sakuda M. Changes in dentofacial morphology in skeletal Class III children treated by a modified maxillary protraction headgear and a chin cup: A longitudinal cephalometric appraisal. Eur J Orthod 1993 Jun;15(3):211-221.

10. Baccetti T, Franchi L, McNamara JA. The cervical vertebral maturation (CVM) method for the assessment of optimal treatment timing in dentofacial orthopaedics. Semin Orthod 2005 Sep;11(3):119-129.

11. Starnbach H, Bayne D, Cleall J, Subtelny JD. Facioskeletal and dental changes resulting from rapid maxillary expansion. Angle Orthod 1966 Apr;36(2):152-164.

12. Chun YS, Jeong SG, Row J, Yang SJ. A new appliance for orthopedic correction of Class III malocclusion. J Clin Orthod 1999;33(12):705-711.

13. Tortop T, Kaygisiz E, Gencer D, Yuksel S, Atalay Z. Modified tandem traction bow appliance compared with facemask therapy in treating Class III malocclusions. Angle Orthod 2014 Jul;84(4):642-648. 